\title{
Asymptotic normality of conditional distribution estimation in the single index model
}

\author{
Diaa Eddine Hamdaoui \\ Laboratory of Stochastic Models, \\ Statistic and Applications, Dr. Tahar \\ Moulay University, Algeria \\ email: diaa-eddine@hotmail.fr

\section{Abbes Rabhi} \\ Mathematics laboratory, Djillali Liabes \\ University of Sidi Bel Abbes, Algeria \\ email: rabhi_abbes@yahoo.fr
}

\author{
Amina Angelika \\ Bouchentouf \\ Mathematics laboratory, Djillali Liabes \\ University of Sidi Bel Abbes, Algeria \\ email: bouchentouf_amina@yahoo.fr

\section{Toufik Guendouzi} \\ Laboratory of Stochastic Models, \\ Statistic and Applications, Dr. Tahar \\ Moulay University, Algeria \\ email: tf.guendouzi@gmail.com
}

\begin{abstract}
This paper deals with the estimation of conditional distribution function based on the single-index model. The asymptotic normality of the conditional distribution estimator is established. Moreover, as an application, the asymptotic $(1-\gamma)$ confidence interval of the conditional distribution function is given for $0<\gamma<1$.
\end{abstract}

\section{Introduction}

The single functional index models have received a considerable attention because of their wide applications in many areas such as economics, medicine, financial econometric and so on. The study of these models has been developed rapidly, see Ait-Saidi et al. (2005, 2008a, 2008b). Recently, Attaoui et

2010 Mathematics Subject Classification: 62G07, 62G5 62G20

Key words and phrases: asymptotic normality, single functional index model, conditional distribution 
al. (2011) investigated the kernel estimator of the conditional density of a scalar response variable $Y$, given a Hilbertian random variable $X$ when the observations are from a single functional index model. The pointwise and the uniform almost complete convergence of the estimator with rates in this model were obtained for independent observations. Furthermore, Ling et al. (2012) obtained the asymptotic normality of the conditional density estimator and the conditional mode estimator for the $\alpha$-mixing dependence functional time series data. Ling et al. (2014) investigated the pointwise almost complete consistency and the uniform almost complete convergence of the kernel estimation with rate for the conditional density in the setting of the $\alpha$-mixing functional data, which extend the i.i.d case in Attaoui et al. (2011) to the dependence setting, the convergence rate of the kernel estimation for the conditional mode was also obtained.

The main contribution of this paper is to establish the asymptotic normality for the estimator of conditional distribution function in the i.i.d. case when the single functional index $\theta$ is fixed. As an application, the asymptotic $(1-\gamma)$ confidence interval for the conditional density function $F(\theta, y, x)$ is presented. The outline of the present paper is as follows. In section 2, we introduce the model as well as basic assumptions that are necessary in deriving the main result of this paper. In section 3 , we state the main result of the paper; the asymptotic normality of the estimator for the conditional distribution function. As an application, the asymptotic $(1-\gamma)$ confidence interval of the conditional distribution function is given for $0<\gamma<1$. Finally, the technical proofs are related to section 4 .

\section{Model and some basic assumptions}

Let $\left\{\left(X_{i}, Y_{i}\right), 1 \leq i \leq n\right\}$ be $n$ random variables, identically distributed as the random pair $(X, Y)$ with values in $\mathcal{H} \times \mathbb{R}$, where $\mathcal{H}$ is a separable real Hilbert space with the norm $\|$.$\| generated by an inner product <., .>$. Under such topological structure and for a fixed functional $\theta$, we suppose that the conditional probability distribution of $Y$ given $\langle X, \theta\rangle=\langle x, \theta\rangle$ exists and is given by

$$
\forall y \in \mathbb{R}, F(\theta, y, x)=\mathbb{P}(Y \leq y \mid<X, \theta>=<x, \theta>
$$

The nonparametric kernel estimator $\widehat{F}(\theta, y, x)$ of $F(\theta, y, x)$ is defined as follows, 


$$
\widehat{F}(\theta, y, x)=\frac{\sum_{i=1}^{n} K\left(h_{K}^{-1}\left(<x-X_{i}, \theta>\right)\right) H\left(h_{H}^{-1}\left(y-Y_{i}\right)\right)}{\sum_{i=1}^{n} K\left(h_{K}^{-1}\left(<x-X_{i}, \theta>\right)\right)},
$$

where $\mathrm{K}$ is a kernel, $\mathrm{H}$ is a cumulative distribution function (cdf) and $h_{K}=$ $h_{K, n}\left(\operatorname{resp}, h_{H}=h_{H, n}\right)$ is a sequence of positive real numbers which goes to zero as $\mathrm{n}$ tends to infinity, and with the convention $0 / 0=0$.

Let, for any $x \in \mathcal{H}, i=1, \ldots, n$ and $y \in \mathbb{R}$

$$
\mathrm{K}_{i}(\theta, x):=\mathrm{K}\left(\mathrm{h}_{\mathrm{K}}^{-1}\left|<x-X_{i}, \theta>\right|\right) \text {, and } \mathrm{H}_{\mathrm{i}}(\mathrm{y}):=\mathrm{H}\left(\mathrm{h}_{\mathrm{H}}^{-1}\left(y-Y_{i}\right)\right) .
$$

We denote by $B_{\theta}(x, h)=\{X \in \mathcal{H} / 0<|<x-X, \theta>|<h\}$ the ball centered at $x$ with radius $h$, let $\mathcal{N}_{x}$ be a fixed neighborhood of $x$ in $\mathcal{H}, S_{R}$ will be a fixed compact subset of $\mathbb{R}$.

Now, we introduce the following basic assumptions that are necessary in deriving the main result of this paper.

(H1) $\mathbb{P}\left(\mathrm{X} \in \mathrm{B}_{\theta}\left(\mathrm{x}, \mathrm{h}_{\mathrm{K}}\right)\right)=: \phi_{\theta, \mathrm{x}}(\mathrm{h})>0, \quad \phi_{\theta, \mathrm{x}}(\mathrm{h}) \rightarrow 0$ as $\mathrm{h} \rightarrow 0$.

(H2) The conditional cumulative distribution $F(\theta, y, x)$ satisfies the Hölder condition, that is:

$\forall\left(y_{1}, y_{2}\right) \in S_{R} \times S_{R}, \forall\left(x_{1}, x_{2}\right) \in \mathcal{N}_{x} \times \mathcal{N}_{x}$.

$\left|F\left(\theta, y_{1}, x_{1}\right)-F\left(\theta, y_{2}, x_{2}\right)\right| \leq C_{\theta, x}\left(\left\|x_{1}-x_{2}\right\|^{b_{1}}+\left|y_{1}-y_{2}\right|^{b_{2}}\right), b_{1}>0, b_{2}>0$.

(H3) For $j=0,1, \mathrm{H}^{(\mathrm{j})}$ satisfies the lipschitz conditions and

$$
m:=\inf _{t \in[0,1]} K(t) H^{\prime}(t)>0,
$$

with

$$
\int H^{\prime}(t) d t=1, \quad \int H^{2}(t) d t<\infty \text { and } \int|t|^{b_{2}} H^{(1)}(t) d t<\infty
$$

(H4) The kernel $\mathrm{K}$ is nonnegative, with compact support $[0,1]$ of class $\mathcal{C}^{1}$ on $[0,1)$ such that $K(1)>0$ and its derivative $K^{\prime}$ exists on $[0,1)$ and $\mathrm{K}^{\prime}(\mathrm{t})<0$.

(H5) For all $u \in[0,1], \lim _{h \rightarrow 0} \frac{\phi_{\theta, x}(u h)}{\phi_{\theta, x}(h)}=\lim _{h \rightarrow 0} \xi_{h}^{\theta, x}(u)=\xi_{0}^{\theta, x}(u)$. 
(H6) The bandwidth $h_{H}$ satisfies,

(i) $\frac{\log n}{\mathrm{n} \phi_{\theta, x}\left(h_{K}\right)} \rightarrow 0$, as $n \rightarrow \infty$.

(ii) $n h_{H}^{2} \phi_{\theta, x}^{2}\left(h_{K}\right) \longrightarrow \infty$, and $\frac{n h_{H}^{3} \phi_{\theta, x}\left(h_{K}\right)}{\log ^{2} n} \longrightarrow \infty$ as $n \rightarrow \infty$.

(iii) $\operatorname{nh}_{\mathrm{H}}^{2} \phi_{\theta, x}^{3}\left(h_{\mathrm{K}}\right) \longrightarrow 0$, as $\mathrm{n} \rightarrow \infty$.

(i) $\frac{\phi_{\theta, x}(\mathrm{~h})}{\mathrm{n}}+\phi_{\mathrm{x}}(\mathrm{h})=\mathcal{O}\left(\frac{1}{\mathrm{n}}\right)$.

(ii) $\sqrt{n \phi_{\theta, x}(h)} \rightarrow 0$ as $n \rightarrow \infty$.

Comments on the assumptions. Assumption (H1) is the same as one given in Ferraty et al. (2005). Assumption (H2) is a regularity conditions which characterize the functional space of our model and is needed to evaluate the bias term of our asymptotic results. Assumptions (H3) and (H5) and (H6) are technical conditions for the proofs. Assumptions (H4) is classical in functional estimation for finite or infinite dimension spaces.

Remark 1 Assumption (H5) is known as (for small h) the "concentration assumption acting on the distribution of $\mathrm{X}$ " in infinite dimensional spaces.

The function $\xi_{h}^{x}(\cdot)$ intervening in assumption (H9) is increasing for all fixed $h$. Its pointwise limit $\xi_{0}^{\mathrm{x}}(\cdot)$ plays a determinant role. It is possible to specify this function (with $\xi_{0}(\mathrm{u}):=\xi_{0}^{\mathrm{x}}(\mathrm{u})$ in the above examples by:

1. $\xi_{0}(u)=u^{\gamma}$,

2. $\xi_{0}(u)=\delta_{1}(u)$, where $\delta_{1}(\cdot)$ is Dirac function,

3. $\xi_{0}(u)=\mathbf{1}_{] 0,1]}(u)$.

\section{Main results: Asymptotic normality of the esti- mator $\widehat{F}(\theta, y, x)$}

In this part of paper, we give the asymptotic normality of the conditional cumulative distribution function in the single functional index model. The main result is given in the following theorem.

Theorem 1 Under Assumptions (H1)-(H7) we have

$$
\sqrt{\frac{n \phi_{\theta, x}\left(h_{K}\right)}{\sigma^{2}(\theta, y, x)}}(\widehat{F}(\theta, y, x)-F(\theta, y, x)) \stackrel{\mathcal{D}}{\longrightarrow} \mathcal{N}(0,1) .
$$


Where

$$
\sigma^{2}(\theta, y, x)=\frac{C_{2}(\theta, x) F(\theta, y, x)(1-F(\theta, y, x))}{C_{1}^{2}(\theta, x)}
$$

with $\mathrm{C}_{\mathrm{j}}(\theta, \mathrm{x})=\mathrm{K}^{\mathrm{j}}(1)-\int_{0}^{1} \mathrm{sK}^{\prime}(\mathrm{s}) \beta_{\theta, \mathrm{x}}(\mathrm{s}) \mathrm{ds}$ for $\mathrm{j}=1,2, " \stackrel{\mathcal{D}}{\longrightarrow}$ " means the convergence in distribution.

Proof. Consider, for $i=1, \ldots, n$,

$$
\begin{aligned}
\mathrm{K}_{i}(\theta, x) & =\mathrm{K}\left(\mathrm{h}_{\mathrm{K}}^{-1}\left(<x-X_{i}, \theta>\right)\right), \mathrm{H}_{i}(y)=\mathrm{H}\left(\mathrm{h}_{\mathrm{H}}^{-1}\left(y-Y_{i}\right)\right), \\
\widehat{\mathrm{F}}_{\mathrm{N}}(\theta, y, x) & =\frac{1}{\mathrm{n} \mathbb{E}\left(\mathrm{K}_{1}(\theta, x)\right)} \sum_{i=1}^{n} \mathrm{~K}_{i}(\theta, x) \mathrm{H}_{i}(y), \\
\widehat{\mathrm{F}}_{\mathrm{D}}(\theta, x) & =\frac{1}{\mathrm{n} \mathbb{E}\left(\mathrm{K}_{1}(\theta, x)\right)} \sum_{i=1}^{n} K_{i}(\theta, x), \\
\Delta_{i}(x, \theta) & =\frac{K\left(h_{\mathrm{K}}^{-1}\left(<x-X_{i}, \theta>\right)\right)}{\mathbb{E} K_{1}(\theta, x)} .
\end{aligned}
$$

In order to establish the asymptotic normality of $\widehat{F}(\theta, t, x)$ we have to consider the following decomposition

$$
\begin{aligned}
\widehat{F}(\theta, y, x)-F(\theta, y, x)= & \frac{\widehat{F}_{N}(\theta, y, x)}{\widehat{F}_{D}(\theta, x)}-\frac{C_{1}(\theta, x) F(\theta, y, x)}{C_{1}(\theta, x)} \\
= & \frac{1}{\widehat{F}_{D}(\theta, x)}\left(\widehat{F}_{N}(\theta, y, x)-\mathbb{E} \widehat{F}_{N}(\theta, y, x)\right) \\
& -\frac{1}{\widehat{F}_{D}(\theta, x)}\left(C_{1}(\theta, x) F(\theta, y, x)-\widehat{F}_{N}(\theta, y, x)\right) \\
& +\frac{F(\theta, y, x)}{\widehat{F}_{D}(\theta, x)}\left(C_{1}(\theta, x)-\mathbb{E}\left[\widehat{F}_{D}(\theta, x)\right]\right) \\
& -\frac{F(\theta, y, x)}{\widehat{F}_{D}(\theta, x)}\left(\widehat{F}_{D}(\theta, x)-\widehat{E}_{D}(\theta, x)\right) \\
= & \frac{1}{\widehat{F}_{D}(\theta, x)} A_{n}(\theta, y, x)+B_{n}(\theta, y, x)
\end{aligned}
$$

where

$$
A_{n}(\theta, y, x)=\frac{1}{n \mathbb{E} K_{1}(x, \theta)} \sum_{i=1}^{n}\left\{\left(H_{i}(y)-F(\theta, y, x)\right) K_{i}(\theta, x)\right.
$$




$$
\left.-\mathbb{E}\left[\left(H_{i}(y)-F(\theta, y, x)\right) K_{i}(\theta, x)\right]\right\}=\frac{1}{n \mathbb{E} K_{1}(x, \theta)} \sum_{i=1}^{n} N_{i}(\theta, y, x),
$$

and

$$
N_{i}(\theta, y, x)=\left(H_{i}(y)-F(\theta, y, x)\right) K_{i}(\theta, x)-\mathbb{E}\left[\left(H_{i}(y)-F(\theta, y, x)\right) K_{i}(\theta, x)\right] .
$$

It follows that,

$$
\begin{aligned}
n \phi_{\theta, x}\left(h_{k}\right) \operatorname{Var}\left(A_{n}(\theta, t, x)\right)= & \frac{\phi_{\theta, x}\left(h_{k}\right)}{\mathbb{E}^{2} K_{1}(x, \theta)} \operatorname{Var}\left(N_{1}\right) \\
& +\frac{\phi_{\theta, x}\left(h_{k}\right)}{n \mathbb{E}^{2} K_{1}(x, \theta)} \sum_{|i-j|>0}^{n} \sum_{n} \operatorname{Cov}\left(N_{i}, N_{j}\right) \\
= & V_{n}(\theta, t, x)
\end{aligned}
$$

Then, the rest of the proof is based on the following Lemmas

Lemma 1 Under hypotheses (H1)-(H3), (H5) and (H7), as $\mathrm{n} \rightarrow \infty$ we have

$$
n \phi_{\theta, x}\left(h_{k}\right) \operatorname{Var}\left(A_{n}(\theta, y, x)\right) \longrightarrow V(\theta, y, x),
$$

where

$$
V(\theta, y, x)=\frac{C_{2}(\theta, x)}{\left(C_{1}(\theta, x)\right)^{2}} F(\theta, y, x)(1-F(\theta, y, x))
$$

Lemma 2 Under hypotheses (H1)-(H3) and (H5)-(H7), as $\mathrm{n} \rightarrow \infty$ we have

$$
\left(\frac{n \phi_{\theta, x}\left(h_{k}\right)}{V(\theta, y, x)}\right)^{1 / 2} A_{n}(\theta, y, x) \stackrel{\mathcal{D}}{\longrightarrow} \mathcal{N}(0,1)
$$

where $\stackrel{\mathcal{D}}{\longrightarrow}$ denotes the convergence in distribution.

Lemma 3 Under assumptions (H1)-(H3) and (H5)-(H7); as $\mathrm{n} \rightarrow \infty$ we have

$$
\sqrt{n \phi_{\theta, x}\left(h_{K}\right)} B_{n}(\theta, y, x) \longrightarrow 0 \text { in Probabilty. }
$$

Now, because the unknown functions $C_{j}(\theta, x)$ and $F(\theta, y, x)$ intervening in the expression of the variance, we need to estimate the quantities $C_{1}(\theta, x)$, $\mathrm{C}_{2}(\theta, x)$ and $\mathrm{F}(\theta, y, x)$, respectively. 
By assumptions $(\mathrm{H} 1)-(\mathrm{H} 4)$ we know that $\boldsymbol{a}_{\mathfrak{j}}(\theta, x)$ can be estimated by $\widehat{\mathrm{C}_{\mathfrak{j}}}(\theta, x)$ which is defined as

$$
\widehat{C}_{j}(\theta, x)=\frac{1}{n \widehat{\phi}_{\theta, x}\left(h_{K}\right)} \sum_{i=1}^{n} K_{i}^{j}(\theta, x), j=1,2
$$

where

$$
\widehat{\phi}_{\theta, x}\left(h_{K}\right)=\frac{1}{n} \sum_{i=1}^{n} I_{\left\{\left|<x-X_{i}, \theta>\right|<h_{k}\right\}} .
$$

By applying the kernel estimator of $F(\theta, y, x)$ given above, the quantity $\sigma^{2}(\theta, x)$ can be estimated finally by:

$$
\widehat{\sigma}^{2}(\theta, x)=\frac{\widehat{C}_{2}(\theta, x) \widehat{F}(\theta, y, x)}{\widehat{C}_{1}^{2}(\theta, x)} \int H^{2}(t) d t
$$

Next, we can derive the following corollary:

Corollary 1 Under assumptions of Theorem 1, we have

$$
\sqrt{\frac{n \widehat{\phi}_{\theta, x}\left(h_{K}\right)}{\widehat{\sigma}^{2}(\theta, y, x)}}(\widehat{F}(\theta, y, x)-F(\theta, y, x)) \stackrel{\mathcal{D}}{\longrightarrow} \mathcal{N}(0,1) .
$$

Thus, following this Corollary we can approximate $(1-\gamma)$ confidence interval of $F(\theta, y, x)$ by

$\widehat{F}(\theta, y, x) \pm t_{\gamma / 2} \times \frac{\widehat{\sigma}(\theta, x)}{\sqrt{n \widehat{\phi}_{\theta, x}\left(h_{K}\right)}}$, where $t_{\gamma / 2}$ is the upper $\gamma / 2$ quantile of standard Normal $\mathcal{N}(0,1)$.

\section{Proofs of technical lemmas}

Proof. [Proof of Lemma 1]

Let

$$
\begin{aligned}
V_{n}(\theta, y, x) & =\frac{\phi_{\theta, x}\left(h_{k}\right)}{\mathbb{E}^{2} K_{1}(\theta, x)} \mathbb{E}\left[K_{1}^{2}(\theta, x)\left(H_{1}(y)-F(\theta, y, x)\right)^{2}\right] \\
& =\frac{\phi_{\theta, x}\left(h_{K}\right)}{\mathbb{E}^{2} K_{1}(\theta, x)} \mathbb{E}\left[K_{1}^{2}(\theta, x) \mathbb{E}\left(\left(H_{1}(y)-F(\theta, y, x)\right)^{2} \mid<\theta, X_{1}>\right)\right]
\end{aligned}
$$

Using the definition of conditional variance, we have

$$
\mathbb{E}\left[\left(H\left(h_{H}^{-1}\left(y-Y_{1}\right)\right)-F(\theta, y, x)\right)^{2} \mid<\theta, X_{1}>\right]=J_{1 n}+J_{2 n},
$$

where 


$$
\begin{gathered}
\mathrm{J}_{1 \mathrm{n}}=\operatorname{Var}\left(\mathrm{H}\left(\mathrm{h}_{\mathrm{H}}^{-1}\left(\mathrm{y}-\mathrm{Y}_{1}\right)\right) \mid<\theta, \mathrm{X}_{1}>\right), \\
\text { and } \\
\mathrm{J}_{2 \mathrm{n}}=\left[\mathbb{E}\left(\mathrm{H}\left(\mathrm{h}_{\mathrm{H}}^{-1}\left(\mathrm{y}-\mathrm{Y}_{1}\right)\right) \mid<\theta, \mathrm{X}_{1}>\right)-\mathrm{F}(\theta, y, x)\right]^{2}
\end{gathered}
$$

$\rightsquigarrow$ Concerning J $\mathrm{In}_{1 \mathrm{n}}$. Let

$$
\begin{aligned}
J_{1 n} & =\mathbb{E}\left[H^{2}\left(\frac{y-Y_{1}}{h_{H}}\right) \mid<\theta, x>\right]-\left(\mathbb{E}\left[H\left(\frac{y-Y_{1}}{h_{H}}\right) \mid<\theta, X_{1}>\right]\right)^{2} \\
& =\mathcal{J}_{1}+\mathcal{J}_{2}
\end{aligned}
$$

- By the property of double conditional expectation, we get that

$$
\begin{aligned}
\mathcal{J}_{1} & =\mathbb{E}\left[H^{2}\left(\frac{y-Y_{1}}{h_{H}}\right)\left|<\theta, X_{1}\right\rangle\right] \\
& =\int_{\mathbb{R}} H^{2}\left(\frac{y-v}{h_{H}}\right) d F\left(\theta, v, X_{1}\right) \\
& =\int_{\mathbb{R}} H^{2}(t) d F\left(\theta, y-h_{H} t, X_{1}\right) .
\end{aligned}
$$

On the other hand, by integrating by part and under assumption (H3), we have

$$
\begin{aligned}
\mathcal{J}_{1}= & \int_{\mathbb{R}} 2 H(t) H^{\prime}(t) F\left(\theta, y-h_{H} t, X_{1}\right) d u \\
= & \int_{\mathbb{R}} 2 H(t) H^{\prime}(t)\left(F\left(\theta, y-h_{H} t, X_{1}\right)-F(\theta, y, x)\right) d u \\
& +\int_{\mathbb{R}} 2 H(t) H^{\prime}(t) F(\theta, y, x) d u .
\end{aligned}
$$

Clearly, we have

$$
\int_{\mathbb{R}} 2 H(t) H^{\prime}(t) F(\theta, y, x) d u=\left[H^{2}(t) F(\theta, y, x)\right]_{-\infty}^{+\infty}=F(\theta, y, x),
$$

thus

$$
\int_{\mathbb{R}} H^{2}(t) d F\left(\theta, y-h_{H} t, X_{1}\right)=F(\theta, y, x)+\mathcal{O}\left(h_{K}^{b_{1}}+h_{H}^{b_{2}}\right) .
$$


$\rightsquigarrow$ Concerning $\mathcal{J}_{2}$. Let

$$
\begin{aligned}
I= & \mathbb{E}\left(H_{i}(y) \mid<X_{1}, \theta>\right) \\
\mathbb{E}\left(H\left(\frac{y-Y_{1}}{h_{H}}\right) \mid<X_{1}, \theta>\right)= & \int_{\mathbb{R}} H\left(\frac{y-u}{h_{H}}\right) f\left(\theta, y, X_{1}\right) d u, \\
= & \int_{\mathbb{R}} H\left(\frac{y-u}{h_{H}}\right) d F\left(\theta, y, X_{1}\right), \\
= & \int_{\mathbb{R}} H^{\prime}\left(\frac{y-u}{h_{H}}\right) F\left(\theta, u, X_{1}\right) d u, \\
= & \int_{\mathbb{R}} H^{\prime}(t)\left(F\left(\theta, y-h_{H} t, X_{1}\right)-F(\theta, y, x)\right) d t \\
& +F(\theta, y, x) \int_{\mathbb{R}} H^{\prime}(t) d t .
\end{aligned}
$$

Because $\mathrm{H}^{\prime}$ is a probability density and by hypotheses (H2) and (H3), we can write:

$$
\begin{aligned}
I & \leq C_{x, \theta} \int_{\mathbb{R}} H^{\prime}(t)\left(h_{K}^{b_{1}}+|t|^{b_{2}} h_{H}^{b_{2}}\right) d t+F(\theta, y, x) \\
& =\mathcal{O}\left(h_{\mathrm{K}}^{b_{1}}+h_{\mathrm{H}}^{b_{2}}\right)+F(\theta, y, x) .
\end{aligned}
$$

Finally, by hypothesis (H3) we get

$$
\mathcal{J}_{2} \longrightarrow \mathrm{F}^{2}(\theta, y, x), \text { as } \mathrm{n} \rightarrow \infty \text {. }
$$

The last equality is due to the fact that $\mathrm{H}^{\prime}$ is a probability density, thus we have by hypothesis (H3)

$$
\int_{\mathbb{R}} H^{\prime}(t)\left(F\left(\theta, y-h_{H} t, X_{1}\right)-F(\theta, y, x)\right) d t \leq \int_{\mathbb{R}} H^{\prime}(t)\left(|t|^{b_{2}} h_{H}^{b_{2}}+h_{K}^{b_{1}}\right) d t \underset{n \rightarrow \infty}{\longrightarrow} 0 .
$$

$\rightsquigarrow$ Concerning $\mathrm{J}_{2 n}$.

We have by integration by parts and changing variables

$$
\begin{aligned}
J_{2 n} & =\mathbb{E}\left(H_{1}(y) \mid<\theta, X_{1}>\right) \\
& =\mathbb{E}\left(H\left(\frac{y-Y_{1}}{h_{H}}\right) \mid<\theta, X_{1}>\right) \\
& =\int H\left(\frac{y-v}{h_{H}}\right) f\left(\theta, v, X_{1}\right) d v
\end{aligned}
$$




$$
\begin{aligned}
& =\int H\left(\frac{y-v}{h_{H}}\right) d F\left(\theta, v, X_{1}\right) \\
& =\int H^{\prime}(t) F\left(\theta, y-h_{H} t, X_{1}\right) d t \\
& =F(\theta, y, x) \int H^{\prime}(t) d t+\int H^{\prime}(t)\left(F\left(\theta, y-h_{H} t, x\right)-F(\theta, y, x)\right) d t
\end{aligned}
$$

the last equality is due to the fact that $\mathrm{H}^{\prime}$ is a probability density.

Thus, we have:

$$
J_{2 n}=F(\theta, y, x)+\mathcal{O}\left(h_{K}^{b_{1}}+h_{H}^{b_{2}}\right)
$$

Finally, we obtain that $\mathrm{J}_{2 \mathrm{n}} \underset{\mathrm{n} \rightarrow \infty}{\longrightarrow} 0$.

Meanwhile, by (H1), (H2), (H4) and (H5), it follows that:

$$
\frac{\phi_{\theta, x}\left(h_{K}\right) \mathbb{E} K_{1}^{2}(\theta, x)}{\mathbb{E}^{2} K_{1}(\theta, x)} \underset{n \rightarrow \infty}{\longrightarrow} \frac{C_{2}(\theta, x)}{\left(C_{1}(\theta, x)\right)^{2}},
$$

Then, by combining equations (5)-(10), it leads to

$$
V_{n}(\theta, y, x) \underset{n \rightarrow \infty}{\longrightarrow} \frac{C_{2}(\theta, x)}{\left(C_{1}(\theta, x)\right)^{2}} F(\theta, y, x)(1-F(\theta, y, x)) .
$$

Proof. [Proof of Lemma 2]

We will establish the asymptotic normality of $A_{n}(\theta, t, x)$ suitably normalized.

We have

$$
\begin{aligned}
\sqrt{n \phi_{\theta, x}\left(h_{K}\right)} A_{n}(\theta, y, x) & =\frac{\sqrt{n \phi_{\theta, x}\left(h_{K}\right)}}{n \mathbb{E} K_{1}(\theta, x)} \sum_{i=1}^{n} N_{i}(\theta, y, x) \\
& =\frac{\sqrt{\phi_{\theta, x}\left(h_{K}\right)}}{\sqrt{n} \mathbb{E} K_{1}(\theta, x)} \sum_{i=1}^{n} N_{i}(\theta, y, x) \\
& =\frac{1}{\sqrt{n}} \sum_{i=1}^{n} \Xi_{i}(\theta, y, x)=\frac{1}{\sqrt{n}} S_{n}
\end{aligned}
$$

Now, we can write,

$$
\Xi_{i}=\frac{\sqrt{\phi_{\theta, x}\left(h_{K}\right)}}{\mathbb{E K}_{1}(\theta, x)} N_{i}
$$


Thus

$$
\operatorname{Var}\left(\Xi_{i}\right)=\frac{\phi_{\theta, x}\left(h_{K}\right)}{\mathbb{E}^{2} K_{1}(\theta, x)} \operatorname{Var}\left(N_{i}\right)=V_{n}(\theta, y, x) .
$$

Note that by (11), we have $\operatorname{Var}\left(\Xi_{i}\right) \longrightarrow V(\theta, y, x)$ as $n$ goes to infinity.

Obviously, we have

$$
\sqrt{\frac{n \phi_{\theta, x}\left(h_{K}\right)}{V(\theta, y, x)}}\left(A_{n}(\theta, y, x)\right)=(n V(\theta, y, x))^{-1 / 2} S_{n}
$$

Thus, the asymptotic normality of $(n V(\theta, y, x))^{-1 / 2} S_{n}$, is deduced from the following results

$$
\begin{aligned}
&\left|\mathbb{E}\left\{\exp \left(i z n^{-1 / 2} S_{n}\right)\right\}-\prod_{j=0}^{n} \mathbb{E}\left\{\exp \left(i z n^{-1 / 2} \Xi_{j}\right)\right\}\right| \longrightarrow 0, \\
& \frac{1}{n} \sum_{j=0}^{n} \mathbb{E}\left(\Xi_{j}^{2}\right) \longrightarrow V(\theta, y, x), \\
& \frac{1}{n} \sum_{j=0}^{n} \mathbb{E}\left(\Xi_{j}^{2} \mathbf{1}_{\left\{\left|\Xi_{j}\right|>\varepsilon\right.} \sqrt{n V(\theta, y, x)\}}\right) \longrightarrow 0, \text { for every } \varepsilon>0 .
\end{aligned}
$$

While equations (13) and (14) show that the $\Upsilon_{j}$ are asymptotically independent, verifying that the sum of their variances tends to $V(\theta, y, x)$. Expression (15) is the Lindeberg-Feller's condition for a sum of independent terms. Asymptotic normality of $S_{n}$ is a consequence of equations (13)-(15).

- Proof of (13) We make use of Volkonskii and Rozanov's lemma (see the appendix in Masry (2005) and the fact that the process $\left(X_{i}\right)$ is i.i.d. Note that using that $\mathrm{V}_{\mathrm{j}}=\exp \left(i \mathrm{in}^{-1 / 2} \mathrm{~S}_{\mathfrak{n}}\right)$, we have

$$
\left|\mathbb{E}\left\{\exp \left(i z n^{-1 / 2} S_{\mathfrak{n}}\right)\right\}-\prod_{j=0}^{n} \mathbb{E}\left\{\exp \left(i z n^{-1 / 2} \Xi_{j}\right)\right\}\right| \longrightarrow 0
$$

as $\mathrm{n}$ goes to infinity.

- Proof of (14) Note that $\operatorname{Var}\left(S_{n}\right) \longrightarrow V(\theta, y, x)$ by equation (11) and (12) (by the definition of the $\Xi_{i}$ ). Then because

$$
\mathbb{E}\left(S_{n}\right)^{2}=\operatorname{Var}\left(S_{n}\right)=\sum_{j=0}^{n} \operatorname{Var}\left(\Xi_{j}\right),
$$


and, using the same arguments as those previously used in the proof of first term of equation (5), we obtain

$$
\frac{1}{n} \sum_{j=1}^{n} \mathbb{E}\left(\Xi_{j}^{2}\right)=\operatorname{Var}\left(\Xi_{1}\right),
$$

as $\operatorname{Var}\left(\Xi_{1}\right) \longrightarrow \mathrm{V}(\theta, y, x)$.

- Proof of (15) Recall that

$$
\Xi_{j}=\sum_{i=0}^{n} r_{i} .
$$

Finally, to establish (15) it suffices to show that the set

$$
\left\{\left|\Xi_{j}\right|>\varepsilon \sqrt{n V(\theta, y, x)}\right\}
$$

is negligible for $n$ large enough.

By using assumptions (H4) and (H5), we have

$$
\left|\Upsilon_{i}\right| \leq C\left(\phi_{\theta, x}\left(h_{K}\right)\right)^{-1 / 2}
$$

therefore

$$
\left|\Xi_{j}\right| \leq \operatorname{Cn}\left(\phi_{\theta, x}\left(h_{K}\right)\right)^{-1 / 2},
$$

which goes to zero as $n$ goes to infinity.

Since

$$
\left|H_{i}(y)-F(\theta, y, x)\right| \leq 1 \text {. }
$$

Then for $n$ large enough, the set $\left\{\left|\Xi_{j}\right|>\varepsilon(n V(\theta, y, x))^{-1 / 2}\right\}$ becomes empty, this completes the proof and therefore that of the asymptotic normality of $(\mathrm{nV}(\theta, y, x))^{-1 / 2} S_{n}$ and the Lemma 2.

Proof. [Proof of Lemma 3]

We have

$$
\begin{aligned}
\sqrt{n \phi_{\theta, x}\left(h_{K}\right)} B_{n}(\theta, y, x)=\frac{\sqrt{n \phi_{\theta, x}\left(h_{K}\right)}}{\widehat{F}_{D}(\theta, x)}\left\{\widehat{E}_{N}(\theta, y, x)-C_{1}(\theta, x) F(\theta, y, x)\right. \\
\left.+F(\theta, y, x)\left(C_{1}(\theta, x)-\mathbb{E}_{D}(\theta, x)\right)\right\} .
\end{aligned}
$$


Firstly, observe that as $n \rightarrow \infty$

$$
\begin{gathered}
\frac{1}{\phi_{\theta, x}\left(h_{K}\right)} \mathbb{E}\left[K^{l}\left(\frac{<x-X_{i}, \theta>}{h_{K}}\right)\right] \rightarrow C_{l}(\theta, x), \text { for } l=1,2 \\
\mathbb{E}\left[\widehat{F}_{D}(\theta, x)\right] \longrightarrow C_{1}(\theta, x),
\end{gathered}
$$

and

$$
\mathbb{E}\left[\widehat{\mathrm{F}}_{\mathrm{N}}(\theta, y, x)\right] \longrightarrow \mathrm{C}_{1}(\theta, x) \mathrm{F}(\theta, y, x)
$$

can be proved in the same way as in Ezzahrioui and Ould Said (2008) corresponding to their Lemmas 5.1 and 5.2. Then the proofs of (16)-(18) are omitted.

Secondly, making use of (16), (17) and (18), we have as $\mathrm{n} \rightarrow \infty$

$$
\left\{\mathbb{E} \widehat{F}_{N}(\theta, y, x)-C_{1}(\theta, x) F(\theta, y, x)+F(\theta, y, x)\left(C_{1}(\theta, x)-\mathbb{E} \widehat{F}_{D}(\theta, x)\right)\right\} \longrightarrow 0
$$

On other hand

$$
\frac{\sqrt{n \phi_{\theta, x}\left(h_{K}\right)}}{\widehat{F}_{D}(\theta, x)}=\frac{\sqrt{n \phi_{\theta, x}\left(h_{K}\right)} \widehat{F}(\theta, y, x)}{\widehat{F}_{D}(\theta, x) \widehat{F}(\theta, y, x)}=\frac{\sqrt{n \phi_{\theta, x}\left(h_{K}\right)} \widehat{F}(\theta, y, x)}{\widehat{F}_{N}(\theta, y, x)} .
$$

Because $\mathrm{K}(\cdot) \mathrm{H}^{\prime}(\cdot)$ is continuous with support on $[0,1]$, then by hypotheses (H3) and (H4) $\exists \mathrm{m}=\inf _{\mathrm{t} \in[0,1]} \mathrm{K}(\mathrm{t}) \mathrm{H}^{\prime}(\mathrm{t})$ such that

$$
\widehat{\mathrm{F}}_{\mathrm{N}}(\theta, y, x) \geq \frac{\mathrm{m}}{\mathrm{h}_{H} \phi_{\theta, x}\left(\mathrm{~h}_{\mathrm{K}}\right)}
$$

which gives

$$
\frac{n \phi_{\theta, x}\left(h_{K}\right)}{\widehat{F}_{N}(\theta, y, x)} \leq \frac{\sqrt{n h_{H}^{2} \phi_{\theta, x}\left(h_{K}\right)^{3}}}{m}
$$

Finally, using (H6), the proof of Lemma 3 is completed. 


\section{References}

[1] A. Ait Saidi, F. Ferraty, R. Kassa, P. Vieu, Cross-validated estimations in the single functional index model, Statistics, 42, (2008a), 475-494.

[2] A. Ait Saidi, F. Ferraty, R. Kassa, P. Vieu, Choix optimal du paramètre fonctionnel dans le modèle à indice fonctionnel simple, C. R. Acad. Sci. Paris, Ser. I, 346, (2008b), 217-220.

[3] A. Ait Saidi, F. Ferraty, R. Kassa, Single functional index model for time series, Romanian J. Pure $\&$ Applied Mathematics, 50, (2005), 321-330.

[4] S. Attaoui, A. Laksaci, E. Ould-Said, A note on the conditional density estimate in the single functional index model, Statist. Probab. Lett, 81(1), (2011), 45-53.

[5] M. Ezzahrioui, E. Ould-Saïd, Asymptotic results of a nonparametric conditional quantile estimator for functional time series, Comm. Statist. Theory Methods, 37 (16-17), (2008), 2735-2759.

[6] F. Ferraty, A. Rabhi, P. Vieu, Conditional quantiles for functional dependent data with application to the climatic El Ninõ phenomenon, Sankhyã : The Indian Journal of Statistics, Special Issue on Quantile Regression and Related Methods, 67 (2), (2005), 378-399.

[7] N. Ling, Q. Xu, Asymptotic normality of conditional density estimation in the single index model for functional time series data, Statistics $\mathscr{E}$ Probability Letters, 82 (12), (2012), 2235-2243.

[8] Nengxiang Ling, Zhihuan Li, Wenzhi Yang (2014) Conditional Density Estimation in the Single Functional Index Model for a-Mixing Functional Data, Communications in Statistics - Theory and Methods, 43:3, 441-454, DOI: $10.1080 / 03610926.2012 .664236$

[9] E. Masry, Nonparametric regression estimation for dependent functional data: Asymptotic normality. Stoch. Proc. and their Appl, 115, (2005), $155-177$. 